\title{
BreastCore
}

\section{Lymph Node Management in Breast Cancer: A Highly Dynamic Evolution}

\author{
Thorsten Kühn \\ Department of Gynecology and Obstetrics, Klinikum Esslingen, Esslingen, Germany
}

In this issue of BREAST CARE, an interdisciplinary panel of European experts discusses the highly dynamic evolution of lymph node management in breast cancer, including current evidence and controversies. Specific emphasis is placed on the view of the different disciplines involved in the multimodal treatment of breast cancer, whose diverging perspectives may explain many of the ongoing debates. Even though the future will clarify a number of existing controversies, it will also provide new issues of debate. Thus, today's standards in lymph node management will continue to change even further in the future, probably diminishing the importance of surgical interventions and hopefully reducing treatmentrelated side effects as well as allowing for more individualized treatments. As clinical research is an important precondition to clarify current uncertainties, the authors present ongoing trials and highlight future perspectives [1-5]. International cooperation appears indispensable to identify open issues and to coordinate, plan, and execute clinical surgical trials, leading to a more systematic and faster generation of practice-changing data. Following this need, EU-BREAST (EUropean Breast Cancer REsearch Association of Surgical Trialists) is a newly founded association with the aim to strengthen European cooperation in the field of locoregional breast cancer treatment.

During the past century, survival rates for breast cancer have improved remarkably. With the extent of surgical interventions continuously declining, more breast cancer patients survive with a better quality of life. This evolution was mainly caused by increasing knowledge about the tumor biology and the identification of molecular breast cancer subtypes, as well as the subsequent development of new specific and effective drugs. At the same time, the surgery of primary breast lesions was reduced from extended radical mastectomy to breast-conserving therapy (BCT). In addition to that, the introduction of neoadjuvant chemotherapy allowed to further limit the extent of surgery and thus an increased rate of $\mathrm{BCT}$, without jeopardizing disease-free (DFS) or overall survival (OS).
Even though the therapeutic effect of lymph node surgery was already questioned many decades ago, e.g. by the NSABP 04 trial, full axillary lymph node dissection (ALND) with the removal of at least 10 lymph nodes remained a cornerstone of surgical treatment for invasive disease until the late 1990s. While still assuming some benefit for locoregional control, the strongest argument for lymph node surgery became the prognostic impact of the axillary nodal status, which, for a long time, was the most important factor for tailoring adjuvant treatment decisions.

The introduction of sentinel lymph node biopsy (SLNB) replaced ALND as a staging procedure early this century, leaving ALND as a therapeutic measure for sentinel lymph node(SLN)positive patients. In view of the high morbidity associated with ALND, however, its therapeutic value became a focus of interest. Two randomized trials assessed the impact of ALND in patients with clinically occult SLN metastases by establishing an experimental arm omitting ALND after positive SLNB. Due to low event rates, neither trial reached its target accrual but at the same time, no clinical benefit from ALND in a modern multimodal treatment concept could be demonstrated. Omitting ALND in patients with clinically occult SLN involvement is therefore becoming increasingly popular. Critics argue that there is yet no sufficient evidence for the equivalence between the performance of ALND and its omission, and that the accompanying adjuvant strategies (e.g. radiation fields) are not sufficiently defined. Medical oncologists may argue that there is still a diagnostic role for ALND in SLN-positive patients in order to identify patients with a high, clinically occult tumor burden. Epidemiologists, on the other hand, refer to numerous historic trials indicating that lymph nodes may not be able to metastasize and should therefore be entirely neglected. In contrast, radiation oncologists refer to recent randomized trials showing a survival benefit for those patients receiving regional nodal irradiation. In this area of conflict it is clear that the interpretation of existing clinical evidence varies widely, and recommendations for treating $\mathrm{cN} 0 \mathrm{pN} 1(\mathrm{sn})$ patients differ considerably in international

\section{KARGER}

() 2018 S. Karger GmbH, Freiburg

Fax +497614520714
Prof. Dr. med. Thorsten Kühn

Department of Gynecology and Obstetrics

Klinikum Esslingen

Hirschlandstraße 97, 73730 Esslingen, Germany

t.kuehn@ $@$ klinikum-esslingen.de 
guidelines. Ongoing clinical trials will clarify most issues of debate. The presentation of different perspectives by de Boniface et al. [2], however, may help to understand the ongoing debate and to find the best solution for individual patients.

Increasing knowledge about intrinsic subtypes and their specific responsiveness to different drugs modified the selection criteria for adjuvant systemic treatment, rendering the $\mathrm{pN}$ stage less important. While patients with a triple-negative receptor status as well as those with HER2-positive tumors are candidates for chemotherapy independently of the $\mathrm{N}$ stage, patients with luminal A tumors will rarely be recommended cytotoxic treatment even in case of axillary tumor burden. The axillary status, assessed by SLNB, is therefore losing its importance for systemic as well as surgical treatment decisions. On the other hand, recently published data show an advantage for radiotherapy (RT) to the chest wall and/or the regional lymph nodes for all $\mathrm{pN} 1$ patients. Thus, the interest to determine the nodal status is increasing from the view of a radiooncologist. Critics argue that the benefit from RT is small and that the clinical improvement by deescalating surgery outweighs the benefit of RT especially in the era of modern systemic regimens that include targeted therapies and probably compensate for the small benefit of RT found in historic trials. Two ongoing randomized trials (SOUND, INSEMA) compare clinically node-negative patients to undergo SLNB or not. Awaiting the results from these trials, an interdisciplinary team of authors coordinated by Reimer [1] outlines some controversial issues regarding the omission of axillary staging in patients with early breast cancer and makes the reader understand the different perspectives (epidemiologist, surgeon, medical oncologist, radiooncologist).

Neoadjuvant chemotherapy (NACT) becomes increasingly popular, allowing for less extensive breast surgery after good response. Patients who were originally candidates for mastectomy or extensive oncoplastic procedures may be treated by simple wide local excision without jeopardizing survival. Furthermore, the effect of chemotherapy on the tumor, be it in the breast and/or the lymph nodes, is an important prognostic factor with an increasing potential to tailor post-neoadjuvant treatment decisions. Two important issues are discussed with regard to lymph node management in the neoadjuvant approach:

(1) Is SLNB reliable and safe after NACT?

(2) Can the extent of regional treatment be reduced after a good response to NACT?

SLNB after NACT for cN0 patients is widely accepted in the surgical community due to excellent detection rates for the procedure in this patient cohort. Axillary staging after NACT is indispensable for the determination of a histopathologic complete response while the indication for adjuvant RT, on the other hand, is still guided by the upfront $\mathrm{N}$ stage. This 'clash of interests' currently remains unsolved.

The reliability and safety of SLNB after NACT is still debated for patients who initially present with clinically positive nodes and who convert to an ycN0 status. Most guidelines still recommend ALND for these patients, but since complete response rates in the breast and axilla are steadily increasing, overtreatment in the growing cohort of patients with a negative lymph node status after
NACT needs to be avoided. New strategies to reduce the failure rates for post-neoadjuvant axillary staging (by patient selection or technical improvement) are presented and their level of evidence is discussed. It is of utmost clinical importance to examine the potential of neoadjuvant treatment to deescalate locoregional treatment (surgery, RT), and therefore, hypotheses derived from modern retrospective studies and their translation into ongoing and future study concepts are discussed.

Increasing insights into the biology of tumor cells and their dissemination patterns may impact on surgical interventions in the future. Only $50 \%$ of patients with involved but untreated lymph nodes developed nodal disease in the NSABP B-04 trial although adjuvant RT or systemic treatment was omitted. Until today, the biological mechanisms that promote or inhibit tumor progression in lymph nodes are poorly understood. The question whether lymph node metastases themselves have a potential to metastasize is controversially discussed. Data from surgical trials and preliminary results from translational research suggest that lymph node metastases themselves cannot seed distant metastases; large randomized trials that compared regional RT versus no further regional treatment, however, show improved distant DFS and even OS after regional lymph node irradiation. The integration of translational research into future surgical trials is of utmost importance to understand the biology and prognostic significance of lymph node involvement in breast cancer. The identification of lymph node metastases with or without the impact to seed distant metastases may impact future surgical strategies. Peintinger et al. [4] summarize the current insights and future perspectives of translational research regarding the management of lymph nodes in breast cancer.

The use of radioisotopes with or without the application of blue dye is currently regarded as the gold standard for SLN identification. Due to some disadvantages (availability of radioactive tracers, costs, exposure of patients to radioactivity, and logistic inconveniences caused by legal requirements), this standard procedure has recently been challenged by competing technologies. Superparamagnetic iron oxide (SPIO), fluorescence techniques, or non-operative staging procedures (e.g. contrast-enhanced ultrasound) are currently being discussed. In view of the declining role of axillary staging in breast cancer, more cost-intensive procedures, although technically sophisticated, will hardly replace the current standard of care with its high efficacy and safety shown in multiple clinical trials. For middle- and low-income countries, the role and technique of axillary staging must be adjusted to the specific clinical implications and financial resources. Goyal [5] summarizes the new technologies for SLN detection and presents the evidence regarding their success rates. The author discusses the clinical effectiveness of different techniques in terms of costs, intraoperative effort, and handling.

There is hardly an area in the field of cancer treatment that undergoes such rapid and continuous modifications as the surgical approach to lymph nodes in breast cancer. Study results derived from randomized milestone studies are rated differently with regard to their level of evidence and their power to change the existing standards of care. This leads to significant uncertainties and a wide range of recommendations in international guidelines for specific clinical 
situations. One example is the surgical management of patients with clinically occult SLN involvement: Although the data from ACOSOG Z0011, AMAROS, and IBCSG 1-23 have been available for almost one decade now, recommendations for axillary management of patients with clinically occult lymph node involvement vary widely. It is furthermore unclear to which extent the results of these studies can be translated to patient subgroups that do not precisely fulfil the inclusion criteria of published trials or who are underrepresented in these studies. It will take another 5-10 years until the data from confirmatory trials (POSNOC, SENOMAC, INSEMA) will be available to clarify current uncertainties.

Three major obstacles for rapidly implementable clinical research in the field of breast cancer surgery can be identified:

(1) Due to low event rates, many clinical trials do not achieve their target accrual. The study results are thus associated with statistical uncertainties.

(2) Clinical trials are almost exclusively organized on a national basis, rendering the accrual of sufficient patient numbers even more difficult. This may entirely prevent the conduct of clinical trials or lead to an unacceptably long accrual time.

(3) The national organization of clinical trials results in the performance of several parallel, uncoordinated and competing studies with a very similar design.

All these issues make clinical research in the field of locoregional treatment ineffective and prevent the fast availability of practice-changing data.

\section{EU-BREAST}

In order to promote and coordinate clinical trials in the field of breast cancer surgery, EU-BREAST, a nonprofit organization with its seat in Milan was founded by leading European researchers in the field on September 10, 2018. Its founding members strongly believe in the strength of cross-border clinical trials in Europe. Wellorganized breast centers are readily available due to the long history of quality-assured interdisciplinary breast cancer care in Europe, resulting in high-level scientific research so far organized on a national basis.

The main objectives of EU-BREAST are:

- Cooperation between ongoing trials and provision of prospectively planned joint subgroup analyses.

- Prospective coordination of new study designs in order to systematically improve breast cancer surgery according to the clinical need.

- Avoidance of unnecessary overlap of clinical trials.

- Conduct of high-quality cross-border studies in a large European network and the provision of results in a short period of time.

- Acceleration and cost-effectiveness of clinical research.

- Promotion of communication among the European community of breast surgeons.

This issue of BREAST CARE is mainly designed by the founding members of EU-BREAST who invited further experts from different disciplines involved in the multimodal treatment of breast cancer. This will hopefully help to gain new insights into the current debate and to clarify controversial issues. Cross-border cooperation between surgeons and their interdisciplinary partners is indispensable to ensure rapid and effective progress in the treatment of breast cancer.

\section{Disclosure Statement}

No conflicts of interest to declare.

\section{References}

Reimer T, Engel J, Schmidt M, Vrou Offersen B, Smidt ML, Gentilini OD: Is axillary sentinel lymph node biopsy required in patients who undergo primary breast surgery? Breast Care 2018;13:DOI: 10.1159/000491703. de Boniface J, Schmidt M, Engel J, Smidt ML, Vrou Offersen B, Reimer T: What is the best management of cN0pN1(sn) breast cancer patients? Breast Care 2018; 13:DOI: $10.1159 / 000491704$.

3 Kühn T, Classe J-M, Gentilini OD, Tinterri C, Peintinger F, de Boniface J: Current status and future perspectives of axillary management in the neoadjuvant setting. Breast Care 2018;13:DOI: 10.1159/000492437.
4 Peintinger F, Reitsamer R, Smidt ML, Kühn T, Liedtke $\mathrm{C}$ : Lymph nodes in breast cancer - what can we learn from translational research? Breast Care 2018;13:DOI: $10.1159 / 000492435$.

5 Goyal A: New Technologies for Sentinel Lymph Node Detection. Breast Care 2018;13:DOI: 10.1159/000492436. 